\title{
Enhanced computed tomograms provide accurate assessment of local extension and stage of bladder carcinoma
}

\author{
Erich K. Lang, Quan Nguyen, Karl Zhang \\ Departments of Urology and Radiology, The Johns Hopkins University School of Medicine, Baltimore, \\ $M D, U S A$
}

The choice of appropriate treatment modality for bladder carcinomas depends on accurate preoperative assessment of the stage of the neoplasm (1). Both CT and MRI complemented by reconstructions can provide the needed accurate informations (2).

This 52-year old white male presented with a history of gross hematuria, which intensified during the past 3 months. He had prior episodes of gross hematuria, 3 and 5 years ago, which were attributed to benign prostatic hypertrophy and treated first by TURP and later clinically.

The laboratory data at this admission were RBC 4.1 M/CUMM, WBC 9400 /CUMM Hct 36.2\%, $\mathrm{Hb} 12.4 \mathrm{~g} / \mathrm{dL}$, BUN $32 \mathrm{mg} / \mathrm{dL}$, Creatinine $2.3 \mathrm{mg} / \mathrm{dL}$, Albumin $3.2 \mathrm{gm} / \mathrm{dL}$, ALK Ptas $12.7 \mathrm{U} / \mathrm{L}$, AST 7U/L, ALT $22 \mathrm{U} / \mathrm{L}$ Total Protein $7.2 \mathrm{gm} / \mathrm{dL}$ Urine analysis of a voided specimen showed gross hematuria, but no blood clots, > $500 \mathrm{RBC} / \mathrm{HpF}, 40-50 \mathrm{WBC} / \mathrm{HpF}$, 20-30 bacteria / Hpf, spec gravity 1028; urine culture was reported as negative.

Patient was moderately diaphoretic, BP 148/92, PR 84/min, lungs were clear to auscultation, on CXR there was cephalization of flow and basilar congestion, no infiltrates nor nodules were appreciated. The abdomen was negative. There was tenderness to percussion of the left back and flank.

Four phase enhanced computed tomograms through abdomen and pelvis, complemented by coronal, sagital as well as volume reconstructions were performed.

A 28-second delayed arterial phase CT demonstrates an enhancing tumor along the left lateral bladder wall extending through the muscu- laris into the perivesical fat. After a skip zone, hypervascularity is noted both along the anterior and posterior bladder wall reflecting submucosal tumor propagation. Some hypervascular stranding is noted extending from the anterior bladder wall into the space of Retzius and there is some nodular enhancing tumor anterior in the space of Retzius. One and one half to $2.1 \mathrm{~cm}$ enhancing nodes are noted in the left external iliac node chain (Figure-1).

CTs at a slightly lower scan level demonstrate the large intravesical component of the neoplasm, but also show a circumferential stain indicating

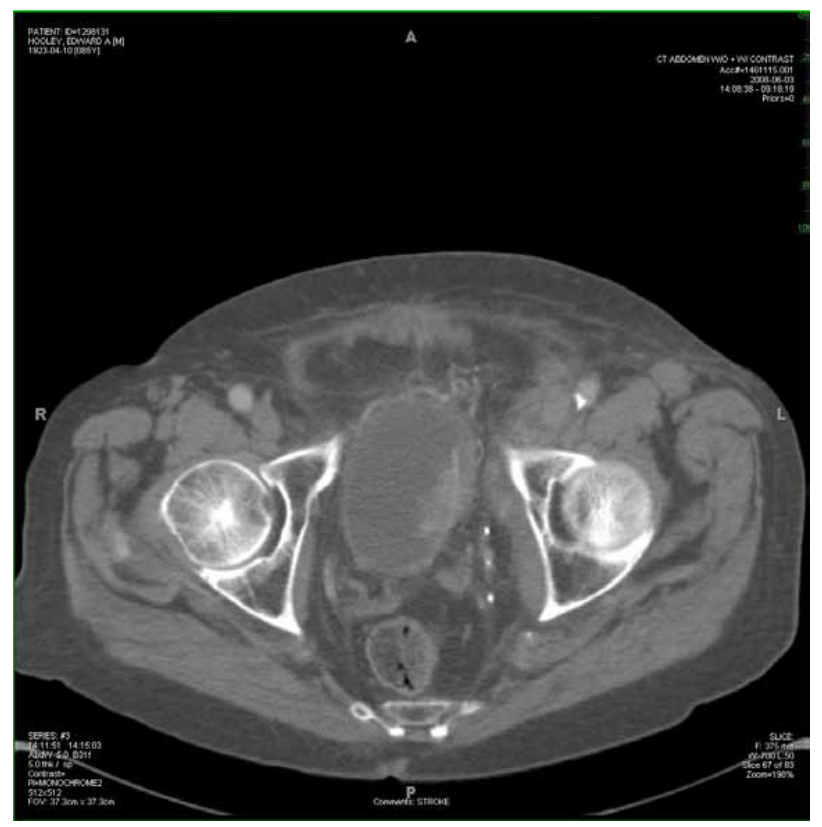

Figure 1 - CT shows extension of enhancing tumor components into the left perivesical space and anteriorly into the space of Retzius. 
submucosal tumor. However, the thickness of the stain also suggests invasion of layers of the muscularis. Huge matted packs of left external and internal iliac nodes are present (Figure-2).

A coronal reconstruction at the anterior pelvic plane level shows contiguous tumor extension into the perivesical fat. Packs of left external iliac nodes $(3.6 \times 2.8 \mathrm{~cm})$ and left para-aortic nodes $(1.6-2.5 \mathrm{~cm}$ size) to the level below the left renal artery are seen (Figure-3).

A coronal reconstruction at the mid-acetabular plane level shows contiguous anterior extension of the neoplasm into the space of Retzius as well as huge packs of nodes of the left external and internal iliac chain to the level of the aortic bifurcation (Figure-4).

Following 2 series of chemotherapy and external radiation therapy to a level of 62gyn, a cystectomy with pelvic and retroperitoneal node dissection was carried out. Histopathology confirmed the accuracy of our CT diagnosis and staging. Though the tumor was significantly reduced in volume, viable tumor cell nests were identified both in the resected perivesical fat as well as in some

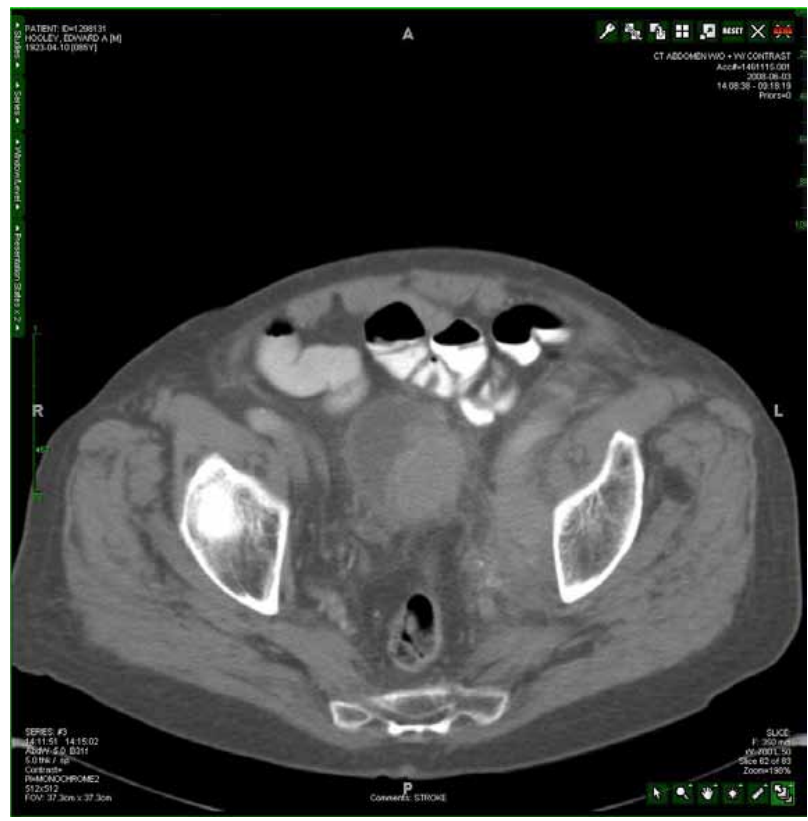

Figure 2 - CT scan shows the massive intravesical tumorcomponent. Note enhancing stain along anterior and posterior circumference of the bladder indicative of submucosal tumor spread with involvement of inner layers of muscularis. common iliac and para-aortic nodes. The patient died 11 months after intervention with evidence of residual neoplastic disease as well as new metastatic disease in lungs and liver.

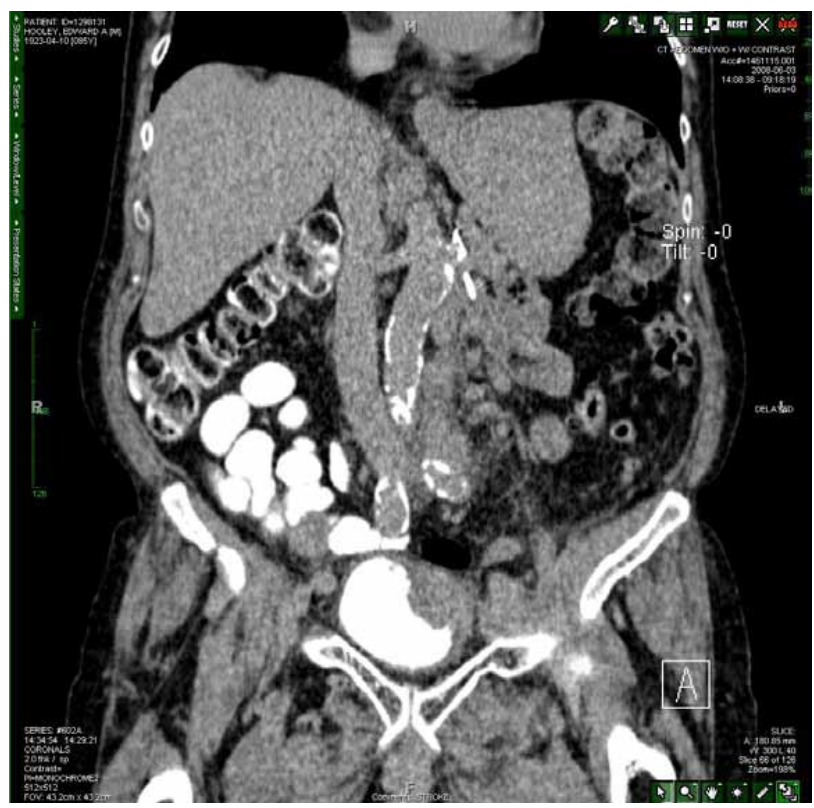

Figure 3 - A coronal reconstruction identifies large para-aortic lymphnode packs extending to the level of the left renal artery.

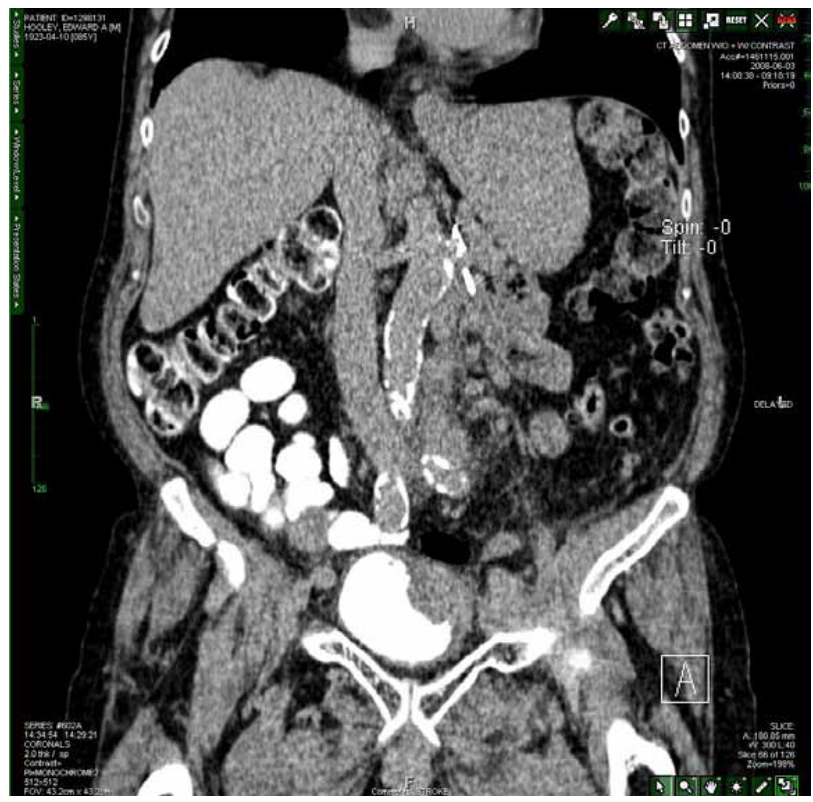

Figure 4 - A coronal reconstruction at the level of the acetabulum demonstrates large lymphnode packs in the left external and common iliac chain to the level of the aortic bifurcation. 


\section{REFERENCES}

1. Fedeli U, Fedewa SA, Ward EM: Treatment of muscle invasive bladder cancer: evidence from the National Cancer Database, 2003 to 2007. J Urol. 2011; 185: 72-8.
2. Barentsz J0, Mitjes JA, Ruijs JH: What's new in bladder imaging? Urol Clin North Am. 1997: 24; 583-91

Correspondence address: Dr. Erich K. Lang Departments of Urology and Radiology SUNY, Downstate Health Science Center 455 Lenox Road Brooklyn, NY, 11203, USA E-mail: erich.lang@downstate.edu 